\title{
The TRAF-interacting protein (TRAIP) is a novel E2F target with peak expression in mitosis
}

\author{
Christophe Chapard ${ }^{1}$, Daniel Hohl ${ }^{1}$ and Marcel Huber ${ }^{1}$ \\ ${ }^{1}$ Service of Dermatology, Lausanne University Hospital, Lausanne, Switzerland \\ Correspondence to: Marcel Huber, email: marcel.huber@chuv.ch \\ Keywords: TRAF-Interacting Protein, TRAIP, E2F transcription factor, promoter regulation, cell cycle \\ Received: October 24, $2014 \quad$ Accepted: November 08, $2014 \quad$ Published: January 02, 2015
}

This is an open-access article distributed under the terms of the Creative Commons Attribution License, which permits unrestricted use, distribution, and reproduction in any medium, provided the original author and source are credited.

\section{ABSTRACT}

The TRAF-interacting protein (TRAIP) is an E3 ubiquitin ligase required for cell proliferation. TRAIP mRNA is downregulated in human keratinocytes after inhibition of the PI3K/AKT/mTOR signaling. Since E2F transcription factors are downstream of PI3K/AKT/mTOR we investigated whether they regulate TRAIP expression. E2F1 expression significantly increased the TRAIP mRNA level in HeLa cells. Reporter assays with the 1400bp 5'-upstream promoter in HeLa cells and human keratinocytes showed that E2F1-, E2F2- and E2F4-induced upregulation of TRAIP expression is mediated by $168 \mathrm{bp}$ upstream of the translation start site. Mutating the E2F binding site within this fragment reduced the E2F1- and E2F2-dependent promoter activities and protein-DNA complex formation in gel shift assays. Abundance of TRAIP mRNA and protein was regulated by the cell cycle with a peak in G2/M. Expression of GFP and TRAIP-GFP demonstrated that TRAIP-GFP protein has a lower steady-state concentration than GFP despite similar mRNA levels. Cycloheximide inhibition experiments indicated that the TRAIP protein has a half-life of around four hours. Therefore, the combination of cell cycle-dependent transcription of the TRAIP gene by E2F and rapid protein degradation leads to cell cycle-dependent expression with a maximum in G2/M. These findings suggest that TRAIP has important functions in mitosis and tumorigenesis.

\section{INTRODUCTION}

The TRAF-interacting protein (TRAIP, TRIP, $\mathrm{RNF206)}$ is a $53-\mathrm{kDa}$ protein that contains at its $\mathrm{N}$-terminal end a 55 amino acids long RING domain, followed by a putative coiled-coil domain and a leucine zipper region [1]. TRAIP undergoes auto-ubiquitination invitro and, by this criterion, can be considered as functional E3 ubiquitin ligase of the RING class [2]. Historically, TRAIP was reported to interact with the tumor necrosis factor (TNF) receptor-associated factors (TRAFs) [2, 3]. Ectopic expression of TRAIP repressed NF- $\mathrm{KB}$ signaling in a RING domain independent manner [2, 3], however, this is most likely a non-physiological function [1]. TRAIP is expressed at low levels in most tissues $[3,4]$ where it is mainly found in the nucleolus of interphase mammalian cells [5]. The nucleolus is a major nuclear substructure with prominent functions in ribosome formation, the control of the cell cycle and stress responses [6].

TRAIP is required for early mice development since homozygous TRAIP knock-out mice die at embryonic day E6.5/7.5 caused by decreased number of proliferative cells and excessive apoptosis [7]. In a screen for cell cycle regulators, Drosophila maternal effect-lethal mutants affecting the 'no poles' (NOPO) gene (CG5140) were identified [8]. This gene encodes a protein with a $\mathrm{N}$-terminal domain showing $47 \%$ sequence identity with the RING domain of human TRAIP [8]. The RING domain of one of the NOPO mutants contained a lysine instead of a glutamic acid at position 11, affecting an amino acid highly conserved in TRAIP homologues. Embryos expressing mutant NOPO undergo mitotic arrest during early embryogenesis showing acentrosomal mitotic spindles as most prominent feature [8].

Recently, it has been reported that NOPO/ TRAIP interacts with DNA polymerase $\eta$ which facilitates translesional synthesis after DNA damage [9]. Furthermore, TRAIP is a regulator of the spindle assembly checkpoint (SAC) by regulating MAD2 levels at kinetochores [10]. In addition, TRAIP interacts with 
CYLD [11] and Syk [5], two tumor suppressors implicated in the formation of skin appendages tumors such as cylindroma, trichoepithelioma and spiradenoma (CYLD) $[12,13]$ and of melanomas and breast tumors (CYLD and Syk) [14-18].

The TRAIP mRNA level is strongly decreased in primary human epidermal keratinocytes (NHEK) undergoing differentiation induced by high calcium concentration, high cell density or phorbol ester [19], indicating that TRAIP expression is down-regulated when cells exit the cell cycle and undergo differentiation. Inhibition of PI3K or mTOR in proliferating keratinocytes reduced also TRAIP mRNA expression [19]. Knockdown of TRAIP by lentiviral-transduced shRNA in primary keratinocytes resulted in strong inhibition of cell proliferation and a G1/S cell-cycle arrest [19]. These results indicated that TRAIP is implicated in the regulation of cellular proliferation and/or survival.

E2F transcription factors are involved in many ways in the regulation of genes for proliferation, DNA replication, apoptosis and differentiation [20]. E2F DNAbinding sites have been found in the promoters of many genes that are crucial to direct cell cycle progression. Eight proteins (E2F1-E2F8) belong to this family of transcription factors which are subdivided into activators (E2F1-E2F3a), repressor (E2F3b-E2F5) and inhibitors (E2F6-E2F8) [21]. The transcriptional activity of E2F1-
E2F5 is regulated by the retinoblastoma protein $(\mathrm{pRb})$ or the related pocket proteins p107 and p130 [22]. To attain their full transcriptional activity and to bind DNA with high affinity, E2F1-E2F6 are required to heterodimerize with dimerization partner (DP) proteins [23].

In this report we show that TRAIP gene expression is induced by E2F1, E2F2 and E2F4 through an E2F binding site located in the 5 'upstream promoter region close to the transcription start site. TRAIP mRNA and protein expression correlated with a peak in the G2/M phase suggesting that TRAIP has physiological function(s) in $\mathrm{G} 2 / \mathrm{M}$.

\section{RESULTS}

\section{E2F1-dependent upregulation of TRAIP expression is inhibited by the retinoblastoma protein}

Previously, we have shown that inhibitors targeting PI3-Kinase (LY294002) or mTOR (rapamycin) decreased TRAIP gene expression in proliferating keratinocytes [19]. These findings strongly suggested that TRAIP expression was regulated by the PI3K/Akt/mTOR signaling pathway. Since the E2F transcription factors are well known targets
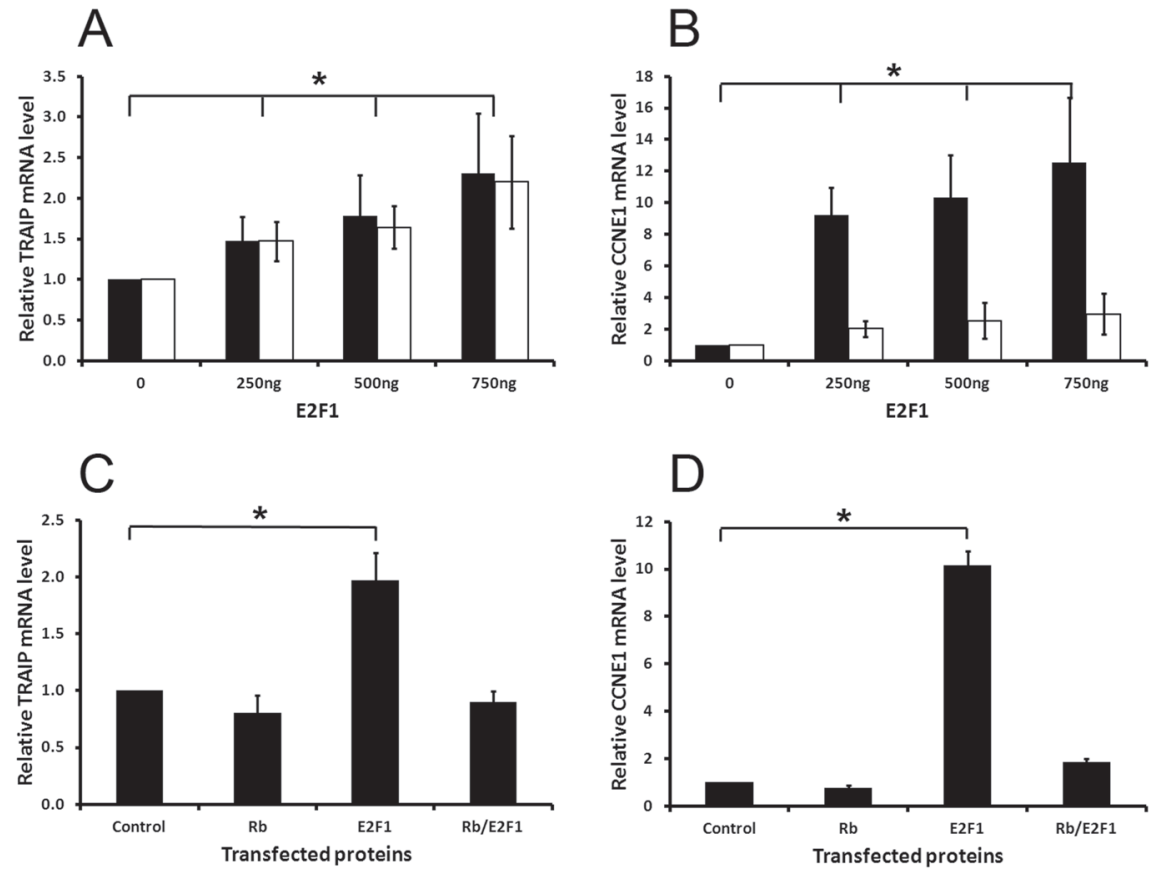

Fig.1: TRAIP mRNA expression is induced by E2F1 but repressed by Rb. Relative mRNA levels of TRAIP (A and C) and CCNE1 (B and D) were measured in HeLa cells 24 (black bars) and 48 (white bars) hours after transfection with the indicated plasmids. In the experiments $\mathrm{C}$ and $\mathrm{D}$ cells were transfected with 400ng pCMV6E2F1 and 600ng pCMV6RB1 plasmid. Control denotes cells transfected with an empty expression vector. RPL13A was used for normalization in quantitative PCR analysis. Results (mean \pm SD) were derived from 4 independent experiments. Statistical significance of differences between sample and control values was calculated by twosided paired t-tests, ${ }^{*} \mathrm{p}<0.05$. 
of this pathway $[24,25]$ and, in addition, rapamycin modulates directly or indirectly the transcriptional activity of E2F [24, 26-28], we sought to determine whether TRAIP is a transcriptional target of E2Fs by transfecting HeLa cells with the vector pCMV6E2F1 expressing E2F1 or an empty control vector. After transfection, cells were cultured in low-serum medium ( $0.25 \%$ fetal bovine serum) in order to reduce endogenous E2F activity and to exclude that serum factors affected the results. RNA was isolated 24 and 48 hours posttransfection and the expression of TRAIP and cyclin E1 (CCNE1) was determined by quantitative RT-PCR analysis. The results demonstrated that TRAIP expression was significantly increased by E2F1 in a dose-dependent manner at 24 and 48 hours posttransfection compared to control cells (Fig. 1A). The transcriptional synthesis of CCNE1, a well-known E2F1 target gene [29, 30], was massively upregulated (Fig. 1B), demonstrating the validity of the experimental system used to detect E2F1 targets in HeLa cells.

Previously, it has been shown that the hypophosphorylated form of the retinoblastoma (pRB1) protein binds to E2F1 leading to the inhibition of its transcriptional activity $[31,32]$. To determine whether the E2F1-dependent activation of TRAIP expression is suppressed by pRB1, HeLa cells were co-transfected with $\mathrm{E} 2 \mathrm{~F} 1$ and $\mathrm{pRB} 1$, and 24 hours post-transfection RNA was isolated for RT-qPCR analysis. The results showed that $\mathrm{pRB} 1$ repressed the E2F1-mediated upregulation of TRAIP mRNA expression (Fig. 1C), similarly to the effect of pRB1 on CCNE1 expression (Fig. 1D). In summary, these experiments demonstrated that the expression of the TRAIP gene is antagonistically regulated by E2F1 and pRB1.

\section{In silico analysis of the 5'-upstream TRAIP promoter}

In silico analysis of the $1.4 \mathrm{kbp} \mathrm{5}$ '-upstream region of the human and mouse TRAIP gene detected two conserved regions with similarity to the consensus E2F binding sequence [33]. In the human gene, one of these binding sites is located downstream of the putative TRAIP transcription start site at the position +16 to +25 whereas the second one is found upstream of the transcription start site at position -628 to -621 (Fig. 2). We therefore investigated whether these two E2F binding sites are involved in the control of TRAIP expression by E2F transcription factors.

\section{Identification of a functional E2F site in the proximal promoter}

Reporter constructs driven by different deletion fragments A-F (Fig. 2) from the $1.4 \mathrm{kbp}$ TRAIP 5'-upstream promoter (-1455/+117; positions +1 and +117 denote the transcription start site and the $G$ residue of the start codon, respectively; data according to ENSEMBL ENSG00000183763) were prepared by cloning into the pGL3-Basic vector. HeLa cells were co-transfected with the different reporter constructs and plasmids expressing E2F family members (E2F1, E2F2, or E2F4). The plasmids pcDNA3 and pGL3-Basic were used as control for expression and reporter constructs, respectively. Firefly luciferase activities were adjusted for transfection efficiency with the co-transfected Renilla luciferase activity. The E2F1-, E2F2-, and E2F4-dependent reporter activities with the promoter fragments A-E were comparable and significantly higher than with the pGL3Basic vector (Fig. 3A-3C). In contrast, the reporter activity

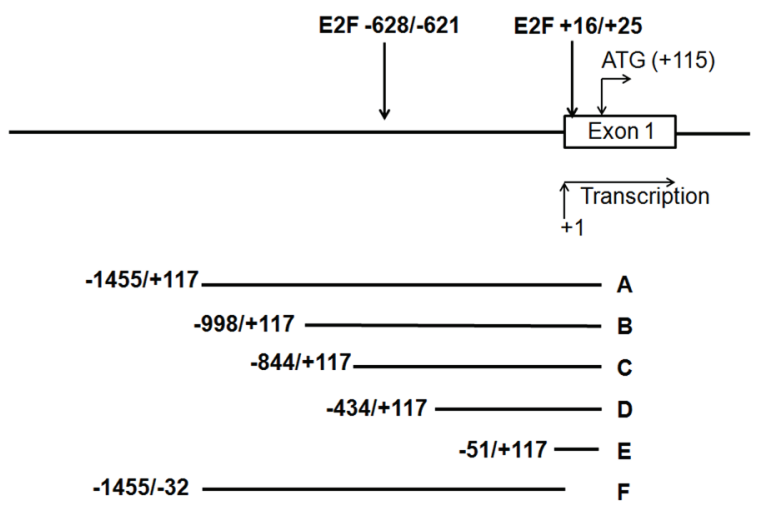

Fig.2: Schematic overview of the 5' promoter region of the human TRAIP gene. The two putative E2F binding sites with their positions relative to the transcription start site are indicated by arrows. The 6 deletion constructs (A-F) which were cloned into the pGL3-Basic luciferase reporter vector are shown below. The transcriptional initiation site is designated as +1 and the nucleotide A of the translation start codon as +115 . 
of fragment $\mathrm{F}$ containing the whole promoter with the exception of $152 \mathrm{bp}$ upstream of the translation initiation codon was indistinguishable from the pGL3-Basic vector with all 3 transcription factors (Fig. 3A-C). These findings argued that the $168 \mathrm{bp}$ region upstream of the translation initiation codon, corresponding to construct E (Fig. 2), contained the functional E2F binding site responsible for E2F1-, E2F2- and E2F4-dependent transactivation of the upstream promoter of the human TRAIP gene in HeLa cells.

Next, we tested the E2F1-dependent reporter activities of the different promoter fragments in primary human keratinocytes (NHEK) cultured in low-calcium medium. The results demonstrated that the fragments A-D had very similar reporter activities which were significantly higher than the basic vector whereas the activity with fragment $\mathrm{F}$ was not increased (Fig. 3D). Taken together, we found that only the $168 \mathrm{bp}$ region $(-51 /+117$, Fig. 2) upstream of the translation initiation

A

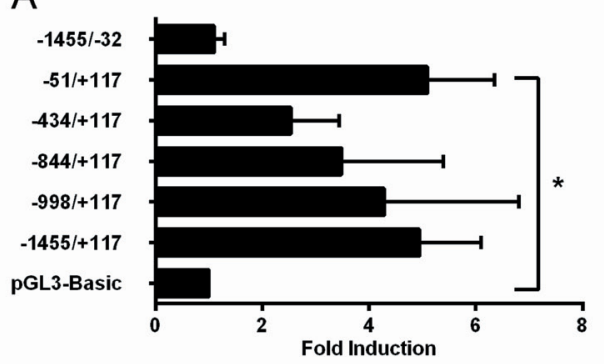

C

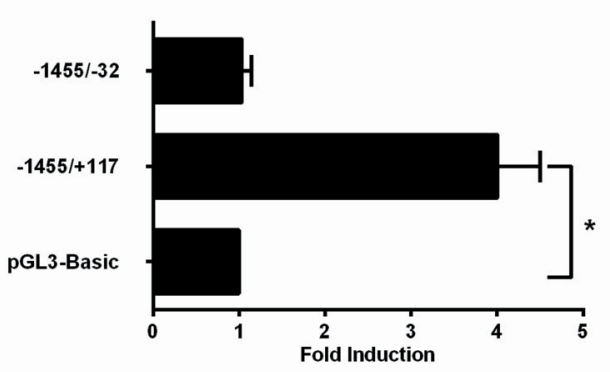

$E$

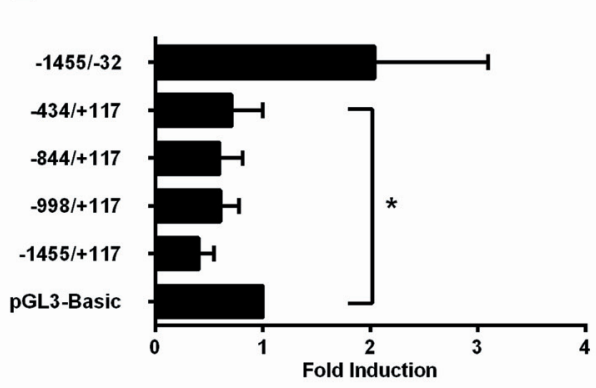

codon was required for the E2F-induced TRAIP gene expression in different cells. This is in good agreement with the in silico identified conserved E2F transcription factor binding site located at position +16 to +25 (Fig. 2). The second putative E2F binding site (-628/-621, Fig. 2) does not seem to be involved in E2F-dependent regulation of TRAIP.

Previously, we have reported that addition of TPA to NHEK cultured in low calcium medium repressed TRAIP expression within 24 hours [19]. Therefore, we investigated whether the promoter activity from the 5'upstream region of the TRAIP gene was modulated by TPA. NHEK were cultured in low calcium medium, transfected with reporter constructs and subsequently treated with $1 \mu \mathrm{M}$ TPA for 24 hours before measuring luciferase activities. The results demonstrated that the reporter activities of the promoter fragments A to D were significantly reduced upon TPA treatment compared to the control vector (Fig. 3E). In contrast, the activity from

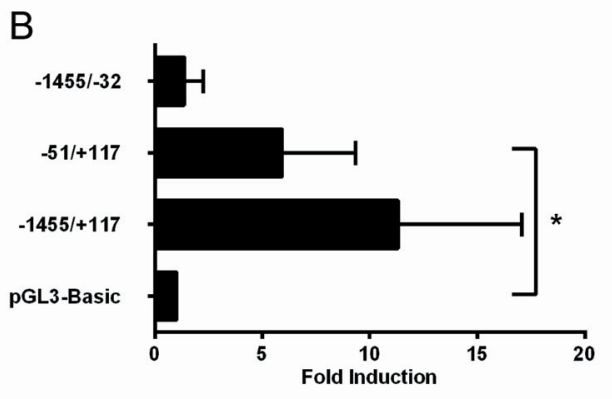

$\mathrm{D}$

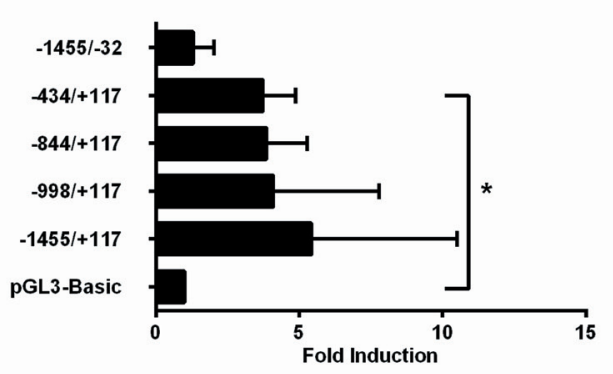

Fig.3: A functional E2F binding site in the TRAIP promoter is located close to the transcription start site. Fold induction of reporter activity in HeLa (A-C) cells or NHEK (D-E) transfected with 750ng pCMV6E2F1 (A, D), pCMV6E2F2 (B), pCMVE2F4 (C), or treated with $1 \mu \mathrm{M}$ TPA (E) after transfection of reporter plasmids. Results are expressed as mean \pm SD from 3-8 experiments performed in duplicates. Statistical significance of differences of reporter activities between promoter plasmids versus pGL3-Basic was calculated by two-sided t-tests, $* \mathrm{p}<0.05$. 
fragment $\mathrm{F}$ was rather increased, albeit not significantly, by TPA. These findings implied that TPA regulated the transcriptional activity of the 5'-upstream part of the TRAIP promoter in a negative manner and localized the TPA-responsive region to the proximal $168 \mathrm{bp}$ fragment of the promoter, similarly as shown above for the E2Fresponse element.

To further prove the binding of E2F transcription factors to the E2F site at +16 to +25 (Fig. 2), the site was mutated from 5'-TTTGGCTC-3' to 5'-TTTATCTC-3' in the full-length promoter (fragment A, Fig. 2). The mutation of the proximal E2F binding site significantly reduced the E2F1- and E2F2-dependent luciferase activities in comparison to the wild type promoter in HeLa cells (Fig. 4A). In electrophoretic mobility shift assays (EMSA) using biotinylated oligonucleotides spanning the proximal E2F binding site (WT) a slower moving protein-DNA complex appeared after incubation with nuclear extracts from proliferating HeLa cells (Fig. 4B, lane 2), which was strongly reduced by competition with 200 -fold molar excess of non-biotinylated WT oligonucleotides (Fig. $4 \mathrm{~B}$, lane 3). No complex was formed after incubation of
HeLa nuclear extracts with biotinylated oligonucleotides containing mutations in the E2F binding site (Mut) (Fig. 4B, lane 4). Furthermore, a 200-fold molar excess of nonbiotinylated Mut oligonucleotides was unable to compete with the biotinylated WT oligonucleotides (Fig. 4B, lane 5). In summary, these data strongly suggest the TRAIP expression is regulated by E2F transcription factors through a functional binding site localized in the proximal region of its 5 'upstream promoter.

\section{Endogenous TRAIP expression is cell cycle regulated with a peak in mitosis}

Since the TRAIP gene is a target of the cell cycle-dependent transcriptional activity of E2Fs we addressed the question whether endogenous TRAIP gene expression is modulated by the cell cycle. HeLa cells were synchronized either in the $\mathrm{G} 0 / \mathrm{G} 1, \mathrm{~S}$ or $\mathrm{G} 2 / \mathrm{M}$ phase by serum starvation, double thymidine block or nocodazole treatment, respectively. Analysis of the cell cycle distributions by fluorescence-activated flow cytometry

A
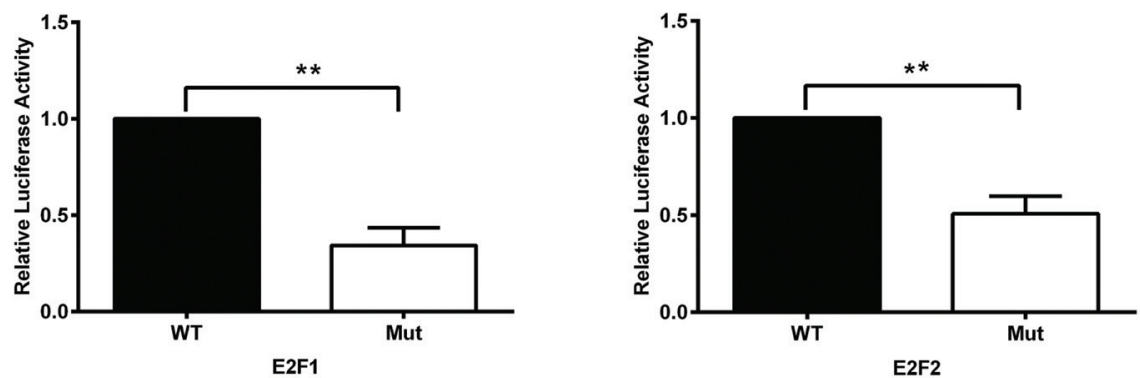

B

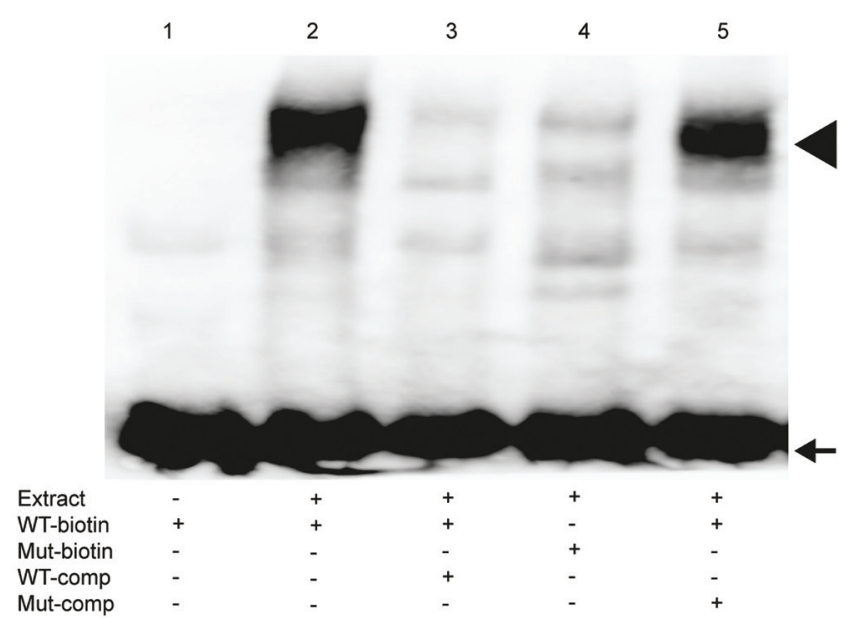

Fig.4: Mutational inactivation of the proximal E2F binding site reduces reporter and EMSA activity. (A) Reporter activity of the normal (WT) and the mutated (Mut) TRAIP promoter with E2F1 (left) and E2F2 (right). Results are expressed as mean \pm SEM from 5 experiments performed in duplicates. Statistical significance of differences of reporter activities between WT and Mut was calculated by two-sided t-tests, ${ }^{* *} \mathrm{p}<0.01$. (B) EMSA experiments with normal (WT) and E2F-mutated (Mut) oligonucleotides using HeLa cell nuclear extracts. Comp; competition with 200-fold molar excess of oligonucleotide added before the biotinylated (biotin) oligonucleotide. Arrow, free oligonucleotide; arrow head, protein-DNA complex. 
analysis of propidium iodide stained cells revealed that a high percentage of cells was arrested in the desired cell cycle phase: G0/G1, $62.8 \pm 2.2 \%$; S, $72.6 \pm 5.3 \%$; G2/M, $80.3 \pm 3.0 \%$; mean \pm SEM, $n=4$. Endogenous TRAIP mRNA levels in the three cell cycle phases were measured by quantitative RT-PCR analysis using four housekeeping genes (RPL13A, GAPDH, RPL3 and B2M) for normalization. Previous experiments reported that their gene expression levels are minimally regulated during the cell cycle [34]. The results showed that the expression of the TRAIP mRNA was significantly higher in G2/M phase cells compared to G0/G1 phase cells with all genes used for normalization (Fig. 5A-D). The expression of TRAIP in the $\mathrm{G} 2 / \mathrm{M}$ phase was also significantly higher than in $\mathrm{S}$ phase after normalization with 3 out of 4 house-keeping genes (RPL13A, RPL3 and B2M). To verify whether the experiment was able to predict correctly the cell cycledependent expression peak of other genes [34], the mRNA levels of E2F1, CCNE1, and CCNB1 were measured in the same synchronized cell populations. As expected, significantly elevated levels of E2F1, CCNE1, and CCNB1 expression were found in the G0/G1, S, and G2/M phase (Fig. 5A-D), respectively, validating our TRAIP data. To further corroborate these data, HeLa cells were blocked in mitosis by nocodazole treatment, then released into normal medium and protein extracts were prepared every three hours. Quantitative Western blot analysis using actin for normalization of protein loading revealed that TRAIP
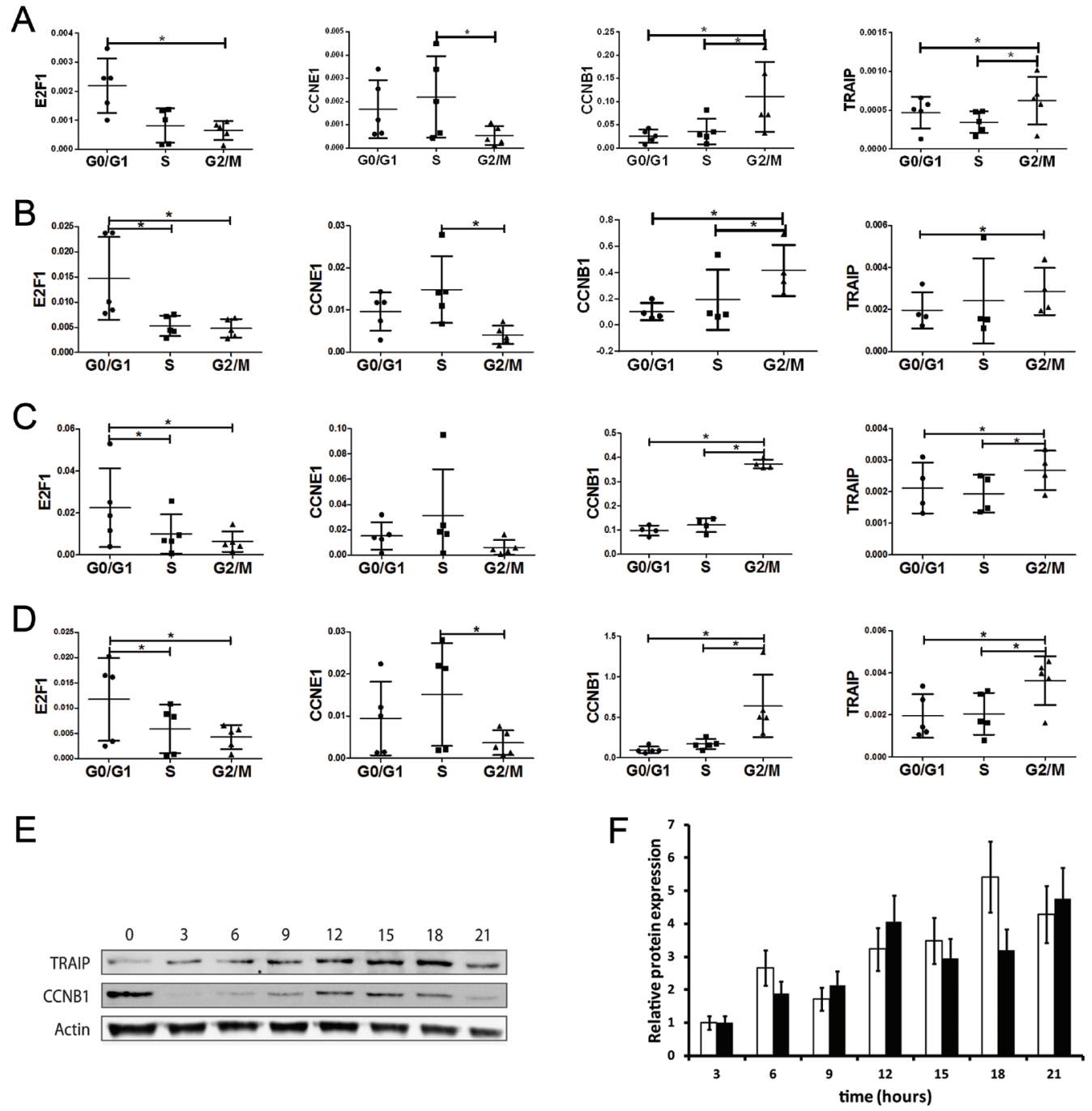

Fig.5: TRAIP is predominantly expressed in the G2/M phase of the cell cycle. RNA was extracted from HeLa cells synchronized in the G0/G1, S and G2/M phase as described in Materials and Methods. Relative gene expressions for E2F1, CCNE1, CCNB1 and TRAIP were normalized either with RPL13A (A), GAPDH (B), RPL3 (C) and B2M (D) by quantitative RT-PCR. Results are depicted as scatter plots of $2^{-\Delta \mathrm{Ct}}$ values (mean $\pm \mathrm{SD}, 4$ independent experiments). Statistical significance of the differences of the $2^{-\Delta \mathrm{Ct}}$ values between the cell cycle phases was calculated by two-sided t-tests; * $\mathrm{p}<0.05$. (E) Western blot analysis of TRAIP and cyclin B1 expression as function of time after release of nocodazole-blocked HeLa cells into normal medium. (F) Protein quantification of cyclin B1 (white bars) and TRAIP (black bars) expression after release from nocodazole block. Results are shown as mean $\pm \mathrm{SD}$ from two experiments using normalization with actin. 
protein expression was cell cycle regulated with a peak at 15 to 18 hours after the release into nocodazole-free medium (Fig. 5E-F). Again, the expression of the G2/M marker cyclin B1 in the same cell extracts followed a kinetic similar to TRAIP protein levels. Thus, the data indicated that TRAIP gene and protein expression are cell cycle regulated with a maximum in the $\mathrm{G} 2 / \mathrm{M}$ phase.

\section{TRAIP protein has a short half-life}

To investigate whether TRAIP protein expression was regulated post-transcriptionally, vectors driving expression of TRAIP-GFP and GFP from a CMV promoter were constructed. TRAIP-GFP was undergoing auto-ubiquitination [2] and localized correctly to the nucleolus [5] as the endogenous protein, indicating that adding GFP at the C-terminal end of TRAIP did not adversely affect the enzymatic activity or the subcellular localization of the fusion protein [10]. HeLa cell lines stably expressing TRAIP-GFP or GFP were obtained by lentiviral infection at equal MOI and hygromycin selection for 11 days. Immunoblot analysis with antiGFP-antibody revealed that the protein level of TRAIPGFP was significantly reduced compared to GFP although their steady-state mRNA levels measured by RT-qPCR using GFP-specific primers were very similar (Fig. 5A). This finding suggested that the TRAIP protein level was post-transcriptionally down-regulated, either due to reduced efficiency of mRNA translation or a higher protein degradation rate. To examine this, $293 \mathrm{~T}$ cells were

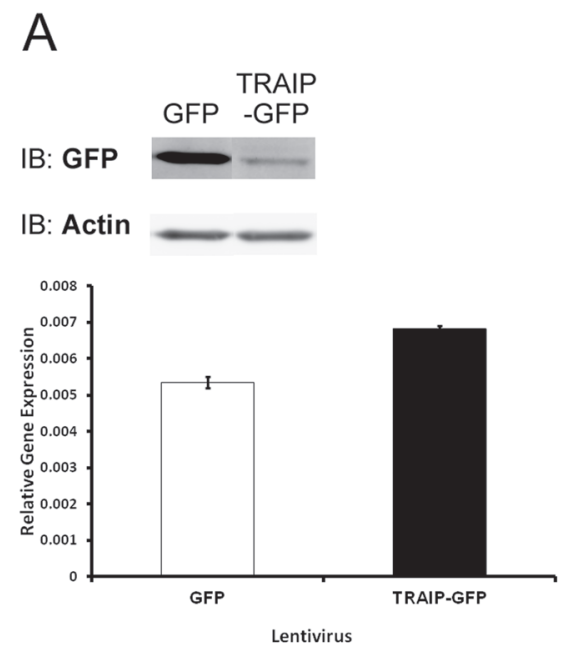

transiently co-transfected with expression vectors for TRAIP-FLAG and GFP and the decrease of TRAIP-FLAG in the first 6 hours of cycloheximide $(50 \mu \mathrm{g} / \mathrm{ml})$ treatment was assessed by immunoblots. GFP or actin were used for normalization since their expressions are more or less stable during the 6 hours of cycloheximide treatment, considering their reported half-lives ( 26 hours for GFP [35], 48 hours for actin [36, 37]). A representative example of the exponential decay of TRAIP-FLAG is shown in Fig. 5B. The calculation [38] from 4 independent experiments revealed a TRAIP half-life (mean \pm SD) of $4 \pm 1.4$ hours or $3.7 \pm 1.6$ hours using normalization with either GFP or actin, respectively. These findings indicated that TRAIP is an unstable protein explaining the different protein steadystate levels of TRAIP-GFP and GFP in stable HeLa cell lines described above.

\section{DISCUSSION}

Cell cycle progression is a complex physiological process coordinated by a multitude of signaling pathways that are regulated by internal and external cues. Experiments using shRNAs, knockout mice and inhibitors showed that the E3 ubiquitin ligase TRAIP is necessary for cellular proliferation [7, 19]. Since TRAIP expression is regulated by the $\mathrm{PI} 3 \mathrm{~K} / \mathrm{AKT} / \mathrm{mTOR}$ pathway [19], which acts upstream of E2F [25-27], we hypothesized that TRAIP is an E2F target gene. Ectopic expression experiments in HeLa showed that TRAIP gene expression is antagonistically regulated by E2F1 and pRB1 (Fig. 1). Indeed, in silico analysis of the 5' upstream region of

B

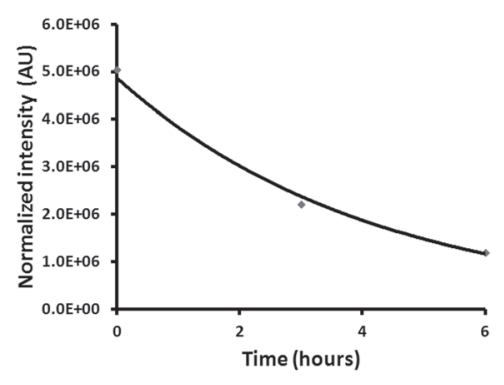

Fig.6: TRAIP protein has a short half-life. (A) Immunoblot with anti-GFP antibody (upper panel) and GFP-specific qRT-PCR (lower panel) analysis in HeLa cells infected at identical MOI with lentivirus expressing GFP or TRAIP-GFP at 11 days post-transduction. Actin served as loading control in immunoblot and the GFP mRNA level was normalized with RPL13A. (B) Decay of TRAIP-FLAG protein after addition of cycloheximide $(50 \mathrm{ug} / \mathrm{ml})$ at time 0 . TRAIP quantity was normalized with actin. One representative example from several similar experiments is shown. 
the human and mouse TRAIP genes revealed two highly conserved putative E2F sites (Fig. 2). Luciferase reporter analysis of the human TRAIP promoter indicated that most, if not all, of the E2F1-, E2F2-, and E2F4-dependent transactivation occurred through the E2F site immediately adjacent to the transcriptional start site (Fig. 3A-E), consistent with the observation that many functional E2F binding sites are located close to the transcription start site [39]. The results from the promoter analysis were not dependent on whether HeLa cells or human keratinocytes were used, indicating that most likely the proximal E2F binding site is important for E2F-dependent TRAIP expression in most tissues. E2F4 was found to activate the TRAIP promoter (Fig. 3C) which might be surprising since this E2F transcription factor is normally thought to function as repressor. However, recent studies indicated that E2F4 can act both as activator and repressor depending on the cellular context and the target gene [40]. The TPA-mediated TRAIP repression in human keratinocytes [19] (Fig. 3E) required only the 168bp fragment containing the proximal E2F site (fragment $\mathrm{E}$, Fig. 2), which would be consistent with data that TPAmediated activation of PKC inhibits AKT, thus decreasing E2F activity, in mouse keratinocytes [41]. The sequence of the proximal E2F binding site (TTTGGCTC) resembles closely the E2F consensus sequence (TTT G/C G/C CGC) [42]. The functional relevance of the proximal E2F binding site in the upregulation of TRAIP expression was further underlined by comparing wild type and mutated E2F site in reporter assays using the full-length promoter (fragment A) and gel shift experiments (Fig. 4). Inspection of large databases from ChIP-on-chip and ChIP-seq experiments, such as ChIPBase [43] and ENCODE (encodeproject.org), and published data [33, 40, 44-46] provided consistent evidence for the binding of E2Fs to the promoter of the human TRAIP gene in close proximity to the transcription start site using different cell lines and experimental conditions. For example, the repressor complexes E2F4/p130 [44] and E2F4/Sin3a/Sin3b [45] were found to bind to the 5' upstream TRAIP promoter using ChIP-on-chip analysis in cell cycle-arrested cells and mouse muscle cells undergoing gene silencing during myogenic differentiation. These findings clearly established that TRAIP gene is a novel direct target of E2F1, E2F2 and E2F4. The transactivation occurs through the proximal promoter region containing a bona fide E2F binding site close to the transcription start site. The finding that several E2F transcription factors modulate TRAIP expression in a comparable manner is not surprising since these transcription factors may have redundant regulatory roles in normal and tumor cells [46].

The distal E2F site (TTGGAGG) at the position $-628 /-621$ (Fig. 2) does not seem to play any role in TRAIP promoter activity under the conditions we have tested. Since the similarity to the consensus sequence is lower compared to the proximal site, E2Fs may bind only with low affinity. However, under other conditions the binding site might still be functional, possibly in connection with other transcription factors and co-regulators, since not all functional E2F binding sites match closely the consensus sequence [33].

Deregulation of E2F activity, through overexpression or gene amplification [47-49], or loss of $\mathrm{pRB} 1$, through viral proteins or mutations, are frequent events causing different types of human tumors. Since TRAIP gene expression is under control of E2F/pRB1, an increase in $\mathrm{E} 2 \mathrm{~F}$ transactivation or $\mathrm{pRB} 1$ inactivation in tumors could augment the TRAIP level. Mice in which all 3 members of the retinoblastoma family (RB1, p107 and p130) were ablated in liver tissue showed increased Traip expression in hepatocellular carcinoma compared to normal tissue [50]. Analysis of gene expression in tamoxifen-treated $\mathrm{Rb}^{\mathrm{F} / \mathrm{F}}$; K14CreER ${ }^{\mathrm{TM}}$; E2F1-/- mouse skin showed a strong decrease in Traip mRNA compared to normal skin [51]. Thus, these in vivo results fit well with our in vitro data showing antagonistically regulation of TRAIP by E2F1 and RB1. Recent work showed that TRAIP expression is increased in breast epithelial cell lines [5], in breast tumors [52], and in basal cell carcinomas (BCC) [19] with a concomitant decrease in CYLD expression $[16,53]$, a tumor suppressor interacting with TRAIP [11]. It has been demonstrated in different cultured mammalian cells and Drosophila that activation of the Hedgehog pathway, a hallmark of BCCs [54], leads to the upregulation of cyclins and E2F transcription factors [55-58]. Hence, this most likely provides an explanation for the overexpression of TRAIP in BCCs. Interestingly, CYLD delays G1-S transition by suppressing cyclin D1 expression and E2F activity [59, 60], and influences mitosis [61]. How TRAIP and CYLD functionally interact in cell cycle regulation and cancer development remains to be explored.

The expression of E2F transcription factors and their target genes are modulated during the cell cycle. E2F transcription factors not only play an important role in controlling genes in $\mathrm{G} 1 / \mathrm{S}$ phase to coordinate DNA replication, but also regulate genes in $\mathrm{G} 2 / \mathrm{M}$ phase encoding proteins important for mitosis [20]. Consistently, TRAIP mRNA and protein expression levels are regulated during the cell cycle reaching a maximum in the $G 2 / \mathrm{M}$ phase (Fig. 5). This is supported by a genome-wide study of gene expression as function of the cell cycle in HeLa cells which listed TRAIP as a periodically expressed gene with a maximum in G2/M [34]. Since the steady-state amount of mRNAs is the combined outcome of synthesis and degradation, the decline in mRNA level after mitosis exit indicates that degradation may be important for TRAIP mRNA regulation during the cell cycle. In silico analysis of 3'-UTR of TRAIP mRNA did not reveal any AU-rich elements which are often, but not always, linked to rapid mRNA turnover $[62,63]$. Recently, microRNA emerged as important factor regulating mRNA half-life 
[64]. Indeed, bioinformatic analysis of TRAIP mRNA detected several evolutionary conserved microRNA binding sites in its 3-'UTR. A Traip mRNA half-life of approximately 3.3 hours was found in mouse embryonic stem cells using different culture conditions [65] indicating that the Traip mRNA has an intermediate stability. TRAIP protein abundance followed closely mRNA expression with a peak in $\mathrm{G} 2 / \mathrm{M}$ suggesting a strong correlation between mRNA and protein synthesis and degradation. Cycloheximide inhibition experiments demonstrated that TRAIP is a short-lived protein with a half-life around 4 hours although stability analysis using the $\mathrm{N}$-terminal rule $[66,67]$ predicts that it should be a stable protein with a half-life greater than 20 hours (ProtLifePred, http:// protein-n-end-rule.leadhoster.com/). In addition, no good PEST [68] motives (epestfind program in the EMBOSS package, http://emboss.sourceforge.net/) were detected in the TRAIP amino acid sequence.

The two findings that the TRAIP protein is unstable and expressed in a cell cycle-dependent manner as E2F target gene with a peak in the G2/M phase, confer upon TRAIP two properties commonly found for proteins involved in mitosis or cell cycle regulation [69]. During mitosis, numerous molecular factors check for correct mitotic spindle formation and sister chromatid separation to guarantee equal distribution of the genetic material to daughter cells [70]. Interestingly, mitotic defects during syncytial divisions in Drosophila melanogaster embryos harboring RING domain mutants in the NOPO gene, the insect homolog of TRAIP, have been reported [8]. This is consistent with our recent results showing that knockdown of TRAIP in HeLa cells reduced MAD2 recruitment to kinetochores and consequently strongly compromised the activity of the spindle assembly checkpoint, ultimately leading to chromosome alignment and segregation defects [10]. Together with the results reported herein, the conclusion emerges that the TRAIP quantity is tightly regulated at the transcriptional and the posttranslational level to provide high amounts of TRAIP at a specific point during the cell cycle $(G 2 / M)$ where it is physiologically required to ensure the mitotic progression of cells with faithful distribution of chromosomes to daughter cells.

In summary, we report novel biological properties of TRAIP which is an E3 ubiquitin ligase essential for cellular proliferation. We show that the cellular TRAIP level is tightly regulated by E2F-mediated transactivation and rapid protein turnover to precisely deliver high amounts of TRAIP in the G2/M phase, which is correlating well with its reported functions during mitosis. To understand fully the biological functions of TRAIP, the identification of its physiological substrates will be necessary. Since overexpression of TRAIP has been reported in basal cell carcinomas [19], breast cancer [5, 52] and melanomas (unpublished results), targeting TRAIP or its substrates may provide new possibilities for therapeutic interventions in tumors.

\section{MATERIALS AND METHODS}

\section{Cell culture}

HeLa, 293T, and 293FT cells were cultured in 3T3 medium [19]. NHEK from foreskin were cultured until passage 5 as described [19].

\section{Plasmid constructions}

Full-length human TRAIP cDNA was isolated from human keratinocytes by RT-PCR and inserted into the pGEM-T-Easy vector (Promega). All vectors used for the expression of TRAIP and GFP proteins were constructed by PCR amplification and cloning either into pEGFP-N3 (Clontech), pCI (Promega) or pCDH-CMV-MCS-EF1Hygro (BioCat). Promoter fragments from the 5'-upstream region of the human TRAIP gene were isolated by PCR and cloned into the pGL3-Basic vector (Promega). Mutations in the E2F binding site were introduced by overlapping PCR amplification using Platinum Pfx DNA Polymerase (Invitrogen) and primers containing the desired mutations. All constructs were verified by sequence analysis. Expression plasmids were purchased from Open Biosystems (E2F1; E2F4; and pRB1) or Addgene (E2F2, Addgene plasmid 24226).

\section{Lentiviral production, titration, and infection}

Lentiviral particles were produced in 293FT cells using the packaging vectors pMD2.G (Addgene plasmid 12259) and psPAX2 (Addgene plasmid 12260), and titrated as described previously [19]. HeLa cells were infected with lentiviral supernatant in the presence of 6 $\mu \mathrm{g} / \mathrm{ml}$ Polybrene (Millipore) at a multiplicity of infection of 10. Twenty-four hours later, cells were selected for 5 to 7 days with $800 \mu \mathrm{g} / \mathrm{ml}$ hygromycin (Calbiochem) until mock-transduced cells were dead.

\section{Cell cycle synchronization in HeLa cells}

HeLa cells were synchronized in G0 phase by 3 days of serum starvation, in $\mathrm{S}$ phase by double thymidine block ( $15 \mathrm{~h}$ in medium supplemented with $2 \mathrm{mM}$ thymidine, $10 \mathrm{~h}$ release, $15 \mathrm{~h}$ with $2 \mathrm{mM}$ thymidine), and in $\mathrm{M}$ phase by $15 \mathrm{~h}$ treatment with $400 \mathrm{ng} / \mathrm{ml}$ nocodazole. Synchronized cells were stained with propidium iodide and analyzed for cell cycle distribution by flow cytometry as described [19]. 


\section{RNA isolation, synthesis of cDNA and qRT-PCR}

RNA was extracted from cultured cells using the RNeasy Mini Kit (Qiagen) and cDNA was produced using the Primescript RT reagent kit (TakaRa). Quantitative PCR analysis was performed with Power SYBRGreen PCR Mastermix (Applied Biosystems) using Quantitect Primer Assay (Qiagen) primers. Relative gene expression was calculated as described [71].

\section{Luciferase reporter assay}

HeLa cells or normal human epidermal keratinocytes (NHEK) were seeded in 24-well plates (2$3 \times 10^{4}$ cells per well) in $3 \mathrm{~T} 3$ medium or EpiLife medium with growth supplements, respectively [19]. Cells were transfected the following day with 250ng of the promotercontaining plasmids and 750ng of plasmids encoding transcription factors using jetPRIME (PolyPlus; for HeLa cells) or jetPEI (PolyPlus; for NHEK) as recommended. Cells were co-transfected with 20ng pTK-RL expressing Renilla luciferase to control for transfection efficiency. After transfection, HeLa cells were cultured in low-serum medium $(0.25 \%(\mathrm{v} / \mathrm{v})$ fetal bovine serum $)$ and NHEK in EpiLife medium with growth supplements. Luciferase activities were measured 24 hours later using the Dual Luciferase Assay Kit (Promega). Reporter activity was calculated by dividing the firefly with the Renilla luciferase activity. The ratio of reporter activities for the different promoter constructs was normalized to the pGL3-Basic vector, arbitrarily set to 1 (results expressed as fold induction).

\section{Nuclear extracts and electrophoretic mobility shift assay}

Nuclear extracts from HeLa cells were prepared using the Nuclear and Cytoplasmic Extraction Kit (ThermoScientific). Electrophoretic mobility shift assays were carried out using the LightShift chemiluminescence EMSA kit (ThermoScientific) following the manufacturer's protocol. The following 5'-biotinylated double-stranded oligonucleotides were used as probes: WT, 5'-CCCGCTCCAGGAAGTCGTGCTGCGGAGC CAAATTTG-3' (the E2F binding site is underlined) and Mut, 5'-CCCGCTCCAGGAAGTCGTGCTGCGAT CATGCGTTTG-3' (the mutated E2F binding site is underlined). The same oligonucleotides without the biotin were used at 200-fold molar excess for competition experiments. After incubation, the reaction products were resolved on a $5 \%$ polyacrylamide gel in $0.5 \times \mathrm{TBE}$ and transferred to HyBond $\mathrm{N}+$ membrane (Amersham).

\section{Protein extractions and Western blot analysis}

Proteins were extracted from cells by lysis in $1 \%$ SDS/phosphate-buffered saline. The following antibodies were used for immunoblots: GFP (Clontech), actin (Sigma), FLAG (Sigma), and cyclin B1 (Cell Signaling Technology). Signals from immunoblots were captured by LAS4000 and quantified by ImageJ.

\section{Statistical analysis}

Statistical analysis was performed with GraphPad Prism 6 and results with $\mathrm{p}<0.05$ were considered significant.

\section{In silico promoter analysis}

The 5'upstream region of the human and mouse TRAIP genes were analysed by the rVISTA 2.0 program to find transcription factor binding sites that are phylogenetically conserved [72].

\section{ACKNOWLEDGMENTS}

We thank Dr. D. Trono for the psPAX2 and pMD2.G plasmids (J Virol 77, 8957 (2003)) and Dr. K. Helin for the expression plasmid pCMVE2F2 (Mol Cell Biol 16, 1047 (1996)). MH designed and supervised the present study. $\mathrm{CC}$ and $\mathrm{MH}$ performed experiments. $\mathrm{CC}, \mathrm{DH}$ and $\mathrm{MH}$ wrote and discussed the paper.

\section{CONFLICTS OF INTEREST}

The authors declare no conflict of interest.

\section{FUNDING}

This work has been supported by the Swiss National Science Foundation grant 31003A-138416 to MH.

\section{Abbreviations}

B2M, Beta2 microglobulin; CYLD, Cylindromatosis; CCNB1, cyclin B1; CCNE1, cyclin E1; DP, dimerization partner; GAPDH, glyceraldehyde3-phosphate dehydrogenase; GFP, Green Fluorescent Protein; mTOR, mammalian Target of Rapamycin; NHEK, Normal Human Epidermal Keratinocytes; NOPO, Drosophila melanogaster No Poles gene; PI3K, phosphatidylinositide-3 kinase; PKC, protein kinase C; RING, Really Interesting New Gene; pRB1, retinoblastoma protein; RPL13A, 60S ribosomal protein L13a; RPL3, 60S ribosomal protein L3; SYK, spleen 
tyrosine kinase; TRAIP, Tumor necrosis factor receptor associated factor (TRAF)-interacting protein; TPA, 12-O-tetradecanoylphorbol-13-acetate.

\section{REFERENCES}

1. Chapard C, Hohl D and Huber M. The role of the TRAFinteracting protein in proliferation and differentiation. Exp Dermatol. 2012; 21: 321-326.

2. Besse A, Campos AD, Webster WK and Darnay BG. TRAF-interacting protein (TRIP) is a RING-dependent ubiquitin ligase. Biochem Biophys Res Commun. 2007; 359: 660-664.

3. Lee SY and Choi Y. TRAF-interacting protein (TRIP): a novel component of the tumor necrosis factor receptor (TNFR)- and CD30-TRAF signaling complexes that inhibits TRAF2-mediated NF-kappaB activation. J Exp Med. 1997; 185: 1275-1285.

4. Su AIet al. A gene atlas of the mouse and human proteinencoding transcriptomes. Proc Natl Acad Sci U S A. 2004; 101: 6062-6067.

5. Zhou Q and Geahlen RL. The protein-tyrosine kinase Syk interacts with TRAF-interacting protein TRIP in breast epithelial cells. Oncogene. 2009; 28: 1348-1356.

6. Boisvert FM, van Koningsbruggen S, Navascues J and Lamond AI. The multifunctional nucleolus. Nat Rev Mol Cell Biol. 2007; 8: 574-585.

7. Park ES, Choi S, Kim JM, Jeong Y, Choe J, Park CS, Choi $\mathrm{Y}$ and Rho J. Early embryonic lethality caused by targeted disruption of the TRAF-interacting protein (TRIP) gene. Biochem Biophys Res Commun. 2007; 363: 971-977.

8. Merkle JA, Rickmyre JL, Garg A, Loggins EB, Jodoin JN, Lee E, Wu LP and Lee LA. no poles encodes a predicted E3 ubiquitin ligase required for early embryonic development of Drosophila. Development. 2009; 136: 449-459.

9. Wallace HA, Merkle JA, Yu MC, Berg TG, Lee E, Bosco $\mathrm{G}$ and Lee LA. TRIP/NOPO E3 ubiquitin ligase promotes ubiquitylation of DNA polymerase eta. Development. 2014; 141: 1332-1341.

10. Chapard C, Meraldi P, Gleich T, Bachmann D, Hohl D and Huber M. The TRAF-interacting protein (TRAIP) is a regulator of the spindle assembly checkpoint. J Cell Sci. 2014; 127: 5149-5156

11. Regamey A, Hohl D, Liu JW, Roger T, Kogerman P, Toftgard R and Huber M. The tumor suppressor CYLD interacts with TRIP and regulates negatively nuclear factor kappaB activation by tumor necrosis factor. J Exp Med. 2003; 198: 1959-1964.

12. Bignell GRet al. Identification of the familial cylindromatosis tumour-suppressor gene. Nat Genet. 2000; 25: $160-165$.

13. Lee DA, Grossman ME, Schneiderman $\mathrm{P}$ and Celebi JT. Genetics of skin appendage neoplasms and related syndromes. J Med Genet. 2005; 42: 811-819.
14. Bailet O, Fenouille N, Abbe P, Robert G, Rocchi S, Gonthier N, Denoyelle C, Ticchioni M, Ortonne JP, Ballotti $\mathrm{R}$, Deckert $\mathrm{M}$ and Tartare-Deckert S. Spleen tyrosine kinase functions as a tumor suppressor in melanoma cells by inducing senescence-like growth arrest. Cancer Res. 2009; 69: 2748-2756.

15. Coopman PJ, Do MT, Barth M, Bowden ET, Hayes AJ, Basyuk E, Blancato JK, Vezza PR, McLeskey SW, Mangeat $\mathrm{PH}$ and Mueller SC. The Syk tyrosine kinase suppresses malignant growth of human breast cancer cells. Nature. 2000; 406: 742-747.

16. Hutti JE, Shen RR, Abbott DW, Zhou AY, Sprott KM, Asara JM, Hahn WC and Cantley LC. Phosphorylation of the tumor suppressor CYLD by the breast cancer oncogene IKKepsilon promotes cell transformation. Mol Cell. 2009; 34: 461-472.

17. Massoumi R, Kuphal S, Hellerbrand C, Haas B, Wild P, Spruss T, Pfeifer A, Fassler R and Bosserhoff AK. Downregulation of CYLD expression by Snail promotes tumor progression in malignant melanoma. J Exp Med. 2009; 206: 221-232.

18. Moroni M, Soldatenkov V, Zhang L, Zhang Y, Stoica G, Gehan E, Rashidi B, Singh B, Ozdemirli M and Mueller SC. Progressive loss of Syk and abnormal proliferation in breast cancer cells. Cancer Res. 2004; 64: 7346-7354.

19. Almeida S, Ryser S, Obarzanek-Fojt M, Hohl D and Huber M. The TRAF-Interacting Protein (TRIP) Is a Regulator of Keratinocyte Proliferation. J Invest Dermatol. 2011; 131: 349-357.

20. Wong JV, Dong P, Nevins JR, Mathey-Prevot B and You L. Network calisthenics: control of E2F dynamics in cell cycle entry. Cell Cycle. 2011; 10: 3086-3094.

21. Chen HZ, Tsai SY and Leone G. Emerging roles of E2Fs in cancer: an exit from cell cycle control. Nat Rev Cancer. 2009; 9: 785-797.

22. Harbour JW, Luo RX, Dei Santi A, Postigo AA and Dean DC. Cdk phosphorylation triggers sequential intramolecular interactions that progressively block $\mathrm{Rb}$ functions as cells move through G1. Cell. 1999; 98: 859-869.

23. Xanthoulis A and Tiniakos DG. E2F transcription factors and digestive system malignancies: how much do we know? World J Gastroenterol. 2013; 19: 3189-3198.

24. Gao N, Zhang Z, Jiang BH and Shi X. Role of PI3K/AKT/ mTOR signaling in the cell cycle progression of human prostate cancer. Biochem Biophys Res Commun. 2003; 310: 1124-1132.

25. Gorshtein A, Rubinfeld H, Kendler E, Theodoropoulou M, Cerovac V, Stalla GK, Cohen ZR, Hadani M and Shimon I. Mammalian target of rapamycin inhibitors rapamycin and RAD001 (everolimus) induce anti-proliferative effects in GH-secreting pituitary tumor cells in vitro. Endocr Relat Cancer. 2009; 16: 1017-1027.

26. Brennan P, Babbage JW, Thomas G and Cantrell D. p70(s6k) integrates phosphatidylinositol 3-kinase and 
rapamycin-regulated signals for $\mathrm{E} 2 \mathrm{~F}$ regulation in $\mathrm{T}$ lymphocytes. Mol Cell Biol. 1999; 19: 4729-4738.

27. Bruemmer D, Yin F, Liu J, Kiyono T, Fleck E, Van Herle AJ and Law RE. Rapamycin inhibits E2F-dependent expression of minichromosome maintenance proteins in vascular smooth muscle cells. Biochem Biophys Res Commun. 2003; 303: 251-258.

28. Javier AF, Bata-Csorgo Z, Ellis CN, Kang S, Voorhees JJ and Cooper KD. Rapamycin (sirolimus) inhibits proliferating cell nuclear antigen expression and blocks cell cycle in the G1 phase in human keratinocyte stem cells. J Clin Invest. 1997; 99: 2094-2099.

29. Dyson $\mathrm{N}$. The regulation of $\mathrm{E} 2 \mathrm{~F}$ by $\mathrm{pRB}$-family proteins. Genes Dev. 1998; 12: 2245-2262.

30. Helin K. Regulation of cell proliferation by the E2F transcription factors. Curr Opin Genet Dev. 1998; 8: 28-35.

31. Bagchi S, Weinmann $\mathrm{R}$ and Raychaudhuri $\mathrm{P}$. The retinoblastoma protein copurifies with E2F-I, an E1Aregulated inhibitor of the transcription factor E2F. Cell. 1991; 65: 1063-1072.

32. Chellappan SP, Hiebert S, Mudryj M, Horowitz JM and Nevins JR. The E2F transcription factor is a cellular target for the RB protein. Cell. 1991; 65: 1053-1061.

33. Rabinovich A, Jin VX, Rabinovich R, Xu X and Farnham PJ. E2F in vivo binding specificity: comparison of consensus versus nonconsensus binding sites. Genome Res. 2008; 18: 1763-1777.

34. Whitfield ML, Sherlock G, Saldanha AJ, Murray JI, Ball CA, Alexander KE, Matese JC, Perou CM, Hurt MM, Brown PO and Botstein D. Identification of genes periodically expressed in the human cell cycle and their expression in tumors. Mol Biol Cell. 2002; 13: 1977-2000.

35. Corish $\mathrm{P}$ and Tyler-Smith C. Attenuation of green fluorescent protein half-life in mammalian cells. Protein Eng. 1999; 12: 1035-1040.

36. Antecol MH, Darveau A, Sonenberg N and Mukherjee BB. Altered biochemical properties of actin in normal skin fibroblasts from individuals predisposed to dominantly inherited cancers. Cancer Res. 1986; 46: 1867-1873.

37. Cheever TR, Olson EA and Ervasti JM. Axonal regeneration and neuronal function are preserved in motor neurons lacking ss-actin in vivo. PLoS One. 2011; 6: e17768.

38. Belle A, Tanay A, Bitincka L, Shamir R and O'Shea EK. Quantification of protein half-lives in the budding yeast proteome. Proc Natl Acad Sci U S A. 2006; 103: 1300413009 .

39. Kel AE, Kel-Margoulis OV, Farnham PJ, Bartley SM, Wingender E and Zhang MQ. Computer-assisted identification of cell cycle-related genes: new targets for E2F transcription factors. J Mol Biol. 2001; 309: 99-120.

40. Lee BK, Bhinge AA and Iyer VR. Wide-ranging functions of E2F4 in transcriptional activation and repression revealed by genome-wide analysis. Nucleic Acids Res. 2011; 39: 3558-3573.
41. Li L, Sampat K, Hu N, Zakari J and Yuspa SH. Protein kinase $\mathrm{C}$ negatively regulates Akt activity and modifies UVC-induced apoptosis in mouse keratinocytes. J Biol Chem. 2006; 281: 3237-3243.

42. Zheng N, Fraenkel E, Pabo CO and Pavletich NP. Structural basis of DNA recognition by the heterodimeric cell cycle transcription factor E2F-DP. Genes Dev. 1999; 13: 666674.

43. Yang JH, Li JH, Jiang S, Zhou H and Qu LH. ChIPBase: a database for decoding the transcriptional regulation of long non-coding RNA and microRNA genes from ChIP-Seq data. Nucl Acids Res. 2013; 41: D177-187.

44. Cam H, Balciunaite E, Blais A, Spektor A, Scarpulla RC, Young R, Kluger Y and Dynlacht BD. A common set of gene regulatory networks links metabolism and growth inhibition. Mol Cell. 2004; 16: 399-411.

45. van Oevelen C, Wang J, Asp P, Yan Q, Kaelin WG, Jr., Kluger Y and Dynlacht BD. A role for mammalian Sin3 in permanent gene silencing. Mol Cell. 2008; 32: 359-370.

46. Xu X, Bieda M, Jin VX, Rabinovich A, Oberley MJ, Green $\mathrm{R}$ and Farnham PJ. A comprehensive ChIP-chip analysis of E2F1, E2F4, and E2F6 in normal and tumor cells reveals interchangeable roles of E2F family members. Genome Res. 2007; 17: 1550-1561.

47. Nelson MAet al. Increased gene copy number of the transcription factor E2F1 in malignant melanoma. Cancer Biol Ther. 2006; 5: 407-412.

48. Pierce AM, Schneider-Broussard R, Gimenez-Conti IB, Russell JL, Conti CJ and Johnson DG. E2F1 has both oncogenic and tumor-suppressive properties in a transgenic model. Mol Cell Biol. 1999; 19: 6408-6414.

49. Saito M, Helin K, Valentine MB, Griffith BB, Willman CL, Harlow E and Look AT. Amplification of the E2F1 transcription factor gene in the HEL erythroleukemia cell line. Genomics. 1995; 25: 130-138.

50. Viatour Pet al. Notch signaling inhibits hepatocellular carcinoma following inactivation of the RB pathway. J Exp Med. 2011; 208: 1963-1976.

51. Costa C, Santos M, Martinez-Fernandez M, Duenas M, Lorz C, Garcia-Escudero R and Paramio JM. E2F1 loss induces spontaneous tumour development in Rb-deficient epidermis. Oncogene. 2013; 32: 2937-2951.

52. Yang C, Trent S, Ionescu-Tiba V, Lan L, Shioda T, Sgroi D and Schmidt EV. Identification of cyclin D1- and estrogenregulated genes contributing to breast carcinogenesis and progression. Cancer Res. 2006; 66: 11649-11658.

53. Kuphal S, Shaw-Hallgren G, Eberl M, Karrer S, Aberger F, Bosserhoff AK and Massoumi R. GLI1-dependent transcriptional repression of CYLD in basal cell carcinoma. Oncogene. 2011; 30: 4523-4530.

54. Hahn Het al. Mutations of the human homolog of Drosophila patched in the nevoid basal cell carcinoma syndrome. Cell. 1996; 85: 841-851.

55. Bhatia B, Hsieh M, Kenney AM and Nahle Z. Mitogenic 
Sonic hedgehog signaling drives E2F1-dependent lipogenesis in progenitor cells and medulloblastoma. Oncogene. 2011; 30: 410-422.

56. Duman-Scheel M, Weng L, Xin S and Du W. Hedgehog regulates cell growth and proliferation by inducing Cyclin D and Cyclin E. Nature. 2002; 417: 299-304.

57. Kenney AM and Rowitch DH. Sonic hedgehog promotes $\mathrm{G}(1)$ cyclin expression and sustained cell cycle progression in mammalian neuronal precursors. Mol Cell Biol. 2000; 20: 9055-9067.

58. Li F, Duman-Scheel M, Yang D, Du W, Zhang J, Zhao C, Qin L and Xin S. Sonic hedgehog signaling induces vascular smooth muscle cell proliferation via induction of the G1 cyclin-retinoblastoma axis. Arterioscler Thromb Vasc Biol. 2010; 30: 1787-1794.

59. Massoumi R. Ubiquitin chain cleavage: CYLD at work. Trends Biochem Sci. 2010; 35: 392-399.

60. Takami Y, Nakagami H, Morishita R, Katsuya T, Hayashi H, Mori M, Koriyama H, Baba Y, Yasuda O, Rakugi $\mathrm{H}$, Ogihara $\mathrm{T}$ and Kaneda Y. Potential role of CYLD (Cylindromatosis) as a deubiquitinating enzyme in vascular cells. Am J Pathol. 2008; 172: 818-829.

61. Stegmeier F, Sowa ME, Nalepa G, Gygi SP, Harper JW and Elledge SJ. The tumor suppressor CYLD regulates entry into mitosis. Proc Natl Acad Sci U S A. 2007; 104: 88698874 .

62. Chen CY and Shyu AB. AU-rich elements: characterization and importance in mRNA degradation. Trends Biochem Sci. 1995; 20: 465-470.

63. Yang E, van Nimwegen E, Zavolan M, Rajewsky N, Schroeder M, Magnasco M and Darnell JE, Jr. Decay rates of human mRNAs: correlation with functional characteristics and sequence attributes. Genome Res. 2003; 13: 1863-1872.

64. $\mathrm{Hu} \mathrm{W}$ and Coller J. What comes first: translational repression or mRNA degradation? The deepening mystery of microRNA function. Cell Res. 2012; 22: 1322-1324.

65. Sharova LV, Sharov AA, Nedorezov T, Piao Y, Shaik N and Ko MS. Database for mRNA half-life of 19977 genes obtained by DNA microarray analysis of pluripotent and differentiating mouse embryonic stem cells. DNA Res. 2009; 16: 45-58.

66. Bachmair A, Finley D and Varshavsky A. In vivo half-life of a protein is a function of its amino-terminal residue. Science. 1986; 234: 179-186.

67. Varshavsky A. The N-end rule pathway and regulation by proteolysis. Protein Sci. 2011; 20(8): 1298-345.

68. Rechsteiner $\mathrm{M}$ and Rogers SW. PEST sequences and regulation by proteolysis. Trends Biochem Sci. 1996; 21: 267-271.

69. Boisvert FM, Ahmad Y, Gierlinski M, Charriere F, Lamont D, Scott M, Barton G and Lamond AI. A quantitative spatial proteomics analysis of proteome turnover in human cells. Mol Cell Proteomics. 2012; 11: M111.011429.
70. Pines J. Cubism and the cell cycle: the many faces of the APC/C. Nat Rev Mol Cell Biol. 2011; 12: 427-438.

71. Livak KJ and Schmittgen TD. Analysis of relative gene expression data using real-time quantitative PCR and the 2(-Delta Delta C(T)) Method. Methods. 2001; 25: 402-408.

72. Loots GG and Ovcharenko I. rVISTA 2.0: evolutionary analysis of transcription factor binding sites. Nucleic Acids Res. 2004; 32: W217-221. 